\title{
Surface-enhanced Raman spectroscopy for the characterization of Vaccinium myrtillus L. bilberries of the Baltic-Nordic regions
}

\author{
Lina Traksele ${ }^{1} \cdot$ Valentinas Snitka $^{1}$
}

Received: 27 June 2021 / Revised: 4 October 2021 / Accepted: 18 October 2021 / Published online: 27 October 2021

(c) The Author(s) 2021

\begin{abstract}
In this study, an investigation of the wild bilberries (Vaccinium myrtillus L.) of the different Baltic-Nordic regions using surface-enhanced Raman spectroscopy (SERS) combined with principal component analysis (PCA) is presented. The bilberries were collected in Lithuania, Latvia, Finland and Norway. The set of the SERS spectra of the berry extracts (pH 4) were recorded on the silver nanoparticles based SERS substrates. The SERS spectra of the extracts were acquired using $532 \mathrm{~nm}$ laser as an excitation source. The morphology of the SERS substrates was evaluated by scanning electron microscopy (SEM) and the presence of the silver nanoparticles was confirmed by the energy-dispersive X-ray spectroscopy (EDX). The enhancement factor (EF) of the silver SERS substrates was found to be $10^{5}$. It has been shown that a strong fluorescence background, associated with the phenolic compounds found in bilberries, can be subtracted due to the fluorescence-quenching properties of the silver nanoparticles. Therefore, an application of the SERS technique allowed to observe the characteristic peaks of the bilberries and the PCA tool enabled to evaluate the spectral variation across the entire SERS data set. The results presented in this paper show that the SERS technique coupled with PCA chemometric analysis might serve as a complementary method that allows to identify the country of origin of the bilberries based on the spectral differences.
\end{abstract}

Keywords SERS $\cdot$ Raman spectroscopy $\cdot$ Bilberries $\cdot$ Anthocyanin $\cdot$ Vaccinium myrtillus

\section{Introduction}

Over the past decade, Raman spectroscopy and SERS began to compete intensively with traditional food research methods such as high-performance liquid chromatography (HPLC), gas chromatography (GS) and enzyme-linked immunosorbent assay (ELISA) [1, 2]. One of the main reasons for such competition is the potential of the SERS to provide the diverse information about the chemical composition of the materials with high sensitivity and specificity. Such capabilities make SERS technique attractive for the detection of molecular traces in various food matrixes leading to the next level control of food quality and safety. SERS has already been applied for the detection of pesticides [2, 3], antibiotics [4, 5], heavy metals [2, 6] and foodborne pathogens $[7,8]$ in various foods, indicating the versatility of the method. Among the mentioned merits, SERS technique also

Lina Traksele

lina.traksele@ktu.lt

1 Food Institute, Kaunas University of Technology, Radvilenu av.19 C, LT 50254 Kaunas, Lithuania holds an advantage in the research of epigenetic events such as DNA methylation occurring due to the alkylation of various bases [9]. Therefore, SERS-based analytical technology expands the possibilities in the research regarding bioactive food components such as probiotics [10]. This paper discusses an application of the SERS for the investigation of the wild bilberries. Due to their beneficial properties for human health, the wild fruits (blueberries, lingonberries, cherries, etc.) and their products are used for food and medical purposes. Therefore, more attention should be paid for the study of the wild fruits at the molecular level. Usually, different analytical methods are applied to test individual components of the berries [11,12] and plant substances [13]. Meanwhile, SERS technique allows to obtain a complex spectra that consist of common molecular vibrations of all the sample's compounds at the same time. This is important for the initial qualitative assessment of raw materials and products, as the overall spectra also reflects the molecular fingerprints of toxic substances such as mycotoxins [14]. A chemically specific SERS tool also provides an opportunity to observe the delicate changes of the molecular structure of flavonoids (i.e., anthocyanin) [15-17]. 
As an inexpensive technique, SERS demonstrates a significant breakthrough in the field of food authenticity and adulteration [18]. The ability to authenticate various food products is a major concern to the food industry as well as to the consumers. Therefore, the identification of ingredients and the detection of adulterants (authentication), as well as the determination of raw material origin (traceability) is an important issue. For this reason, further studies should involve a wider range of different species of the bilberries, as well as the development of the reference databases with defined parameters which may be considered as useful geographic origin markers. Reference values are required for the confirmation of a specific origin and the identification of an unknown origin [19, 20]. Today, innovative and sensitive spectroscopic methods show a potential to assess the authenticity of the products and to ensure the prevention of incorrect labeling.

One of the main challenges that the manufacturers face today is the synthetic dyes, such as amaranth, added to the berry extracts [21,22]. Such adulteration aims to artificially intensify the color of the berries leading to the false evaluation of the total anthocyanin content by UV-Vis method. Incorrect results are observed because the absorption of the dye occurs at the same wavelength as anthocyanin. Therefore, it becomes impossible to distinguish whether the absorption peak belongs to anthocyanin or adulterant substance. In contrast to UV-Vis, SERS allows to perform the qualitative and quantitative detection of amaranth at the trace level with high specificity, which is determined by the unique molecular vibrations of the dye in the spectral fingerprint region [23].

In this work, we focus on the application of the SERS technique for the investigation of the wild bilberries naturally growing in the different Baltic-Nordic regions. Previous research demonstrates that the composition of anthocyanin as well as other bioactive compounds present in the wild bilberries depend not only on the internal (genotype) but also on external (environmental) factors [24]. Based on these findings, we hypothesized that the SERS approach will allow to distinguish the wild bilberries growing at the different locations by analyzing their spectral data. To achieve the aim, we applied SERS technique for the generation of the berries' spectra and PCA tool for an evaluation of the spectral variation across the entire SERS data set. To the best of our knowledge, the comparison of the wild bilberries of the different Baltic-Nordic regions was not reported before.

\section{Materials and methods}

\section{Materials}

Water, silver nitrate $(\geq 99.0 \%)$, hydrofluoric acid (48 wt. \% in $\left.\mathrm{H}_{2} \mathrm{O}, \geq 99.99 \%\right)$, pentacene (99.0\%), methanol
(HPLC, $\geq 99.0 \%$ ), $\mathrm{HCl}$ (37.0\%), D-fructose ( $\geq 99.0 \%$ ), D-glucose $(99.5 \%)$, and sucrose $(\geq 99.5 \%)$ were purchased from Sigma Aldrich. $\mathrm{ZnSO}_{4} \times 2 \mathrm{H}_{2} \mathrm{O}$ and $\mathrm{K}_{4}\left[\mathrm{Fe}(\mathrm{CN})_{6}\right] \times 3 \mathrm{H}_{2} \mathrm{O}$ were purchased from Eurochemicals. All the reagents used for the experiments were of analytical grade and used without further purification. All the bilberries (Vaccinium myrtillus) were harvested at the same ripening stage and frozen at $-20{ }^{\circ} \mathrm{C}$ without further processing.

\section{Methods}

\section{Preparation of the silver SERS substrates}

The solution of silver precursor was prepared by dissolving $\mathrm{AgNO}_{3}$ in water to the final concentration of $0.1 \mathrm{M}$. The prepared $\mathrm{HF}(24 \%)$ and $\mathrm{AgNO}_{3}(0.1 \mathrm{M})$ solutions were mixed in a ratio of $1: 1(\mathrm{v}: \mathrm{v})$. The silicon wafers were immersed into the reaction solution for $5 \mathrm{~s}$, immediately transferred to the distilled water and finally dried under the nitrogen flow. Morphological characterization of the synthesized silver SERS substrates was carried out using SEM microscope (SEM, Hitachi S-3400 N).

For the determination of the EF of the silver SERS substrates, the monolayer of the pentacene was deposited on the nanostructured SERS surface by vacuum evaporation. The thickness of the monolayer was $1-1.5 \mathrm{~nm}$. The conditions applied for the acquirance of the spectra are provided in the next section.

\section{Preparation of the bilberry extracts for SERS measurements}

$1000 \mathrm{~g}$ of the bilberries was collected in each country. The berries were divided into 3 containers (each container contained $330 \mathrm{~g}$ of the bilberries) and homogenized using a blender. $5 \mathrm{~g}$ of the mashed berries from each container was transferred to the separate flasks and diluted with distilled water to $50 \mathrm{ml}$. The solutions were centrifuged for $2 \mathrm{~min}$ at $4000 \mathrm{rpm}$ to precipitate the insoluble parts of the berries (skin and seeds). $5 \mathrm{ml}$ of each centrifugate was transferred to the flasks and diluted with distilled water to $10 \mathrm{ml} .25 \mu \mathrm{l}$ of the prepared solutions ( $\mathrm{pH}$ 4.06) was dropped on the separate silver SERS substrates. Therefore, three samples of the bilberries were prepared for each country and 20 SERS spectra of the bilberries were recorded at the different points of each sample. The graphical illustration of the preparation of the bilberries samples is presented in Fig. S1 (see supplementary material).

The SERS spectra of the bilberries were recorded using Raman spectrometer (NTEGRA Spectra, NT-MDT Inc.). The objective magnification was $100 \times$, numerical aperture $\mathrm{NA}=1.49$ and a spot diameter of the optical beam focus$500 \mathrm{~nm}$. The excitation source was a laser with $\lambda=532 \mathrm{~nm}$ and optical beam power of $25 \mathrm{~mW}$. The laser power on the 
samples was $0.20 \mathrm{~mW}$. An integration time for the collection of the spectra was set to be $1 \mathrm{~s}$. All the spectra were normalized and averaged using Origin Pro 9.0 software.

\section{Preparation of the bilberries samples for UV-Vis measurements}

The preparation of the berries samples for the analysis of total anthocyanin content was based on the European pharmacopeia protocol [25]. Briefly, $5 \mathrm{~g}$ of the homogenized berries was added to the flask and diluted with $95 \mathrm{ml}$ of methanol. The flask was placed into the ultrasonic bath for $20 \mathrm{~min}$. Then, the extract was filtered and diluted with methanol to $100 \mathrm{ml} .1 \mathrm{ml}$ of the prepared extract solution was transferred to the flask and diluted with acidified methanol $(0.1 \% \mathrm{HCl})$ to $50 \mathrm{ml}$. The prepared solution was analyzed at $528 \mathrm{~nm}$ wavelength using UV-VIS spectrophotometer (Agilent, Cary 60). The total content of anthocyanin was calculated according to the following equation:

Total anthocyanin content $(\% w / w)=\frac{A \times 5000}{718 \times m}$,

where 718 is the absorption of cyaniding-3-glucoside at $528 \mathrm{~nm} ; A$ is the absorbance of the test sample at $528 \mathrm{~nm} ; \mathrm{m}$ is the sample weight, $\mathrm{mg}$.

\section{High-performance liquid chromatography analysis of saccharides}

Preparation of the standard solutions $200 \mathrm{mg}$ of fructose, glucose and sucrose were added to the separate flasks and diluted with water to $100 \mathrm{ml}$. The final concentrations of the saccharides in the solutions were $2 \mathrm{mg} / \mathrm{ml}$.

Preparation of the test solutions $3 \mathrm{~g}$ of the homogenized berries was transferred to the flask and diluted with water to $50 \mathrm{ml}$. The prepared solution was placed into the water bath for $15 \mathrm{~min}$ at $60{ }^{\circ} \mathrm{C}$. Then, the solution was cooled to room temperature and treated with Carrez I (7.20 g of $\mathrm{ZnSO}_{4} \times 2 \mathrm{H}_{2} \mathrm{O}$ diluted with water to $\left.100 \mathrm{ml}\right)$ and Carrez II (3.60 $\mathrm{g}$ of $\mathrm{K}_{4}\left[\mathrm{Fe}(\mathrm{CN})_{6}\right] \times 3 \mathrm{H}_{2} \mathrm{O}$ diluted with water to $100 \mathrm{ml}$ ) solutions. The final transparent solutions were filtered through membrane filters (size of the pores $-0.45 \mu \mathrm{m}$ ) and analyzed by HPLC system (Shimadzu). The content of the saccharides was calculated according to the following equation:

Saccharides content $(\% w / w)=\frac{S_{\mathrm{s}} \times C_{\mathrm{st}} \times V \times 100}{S_{\mathrm{st}} \times 1000 \times m}$,

where $S_{\mathrm{s}}$ is the sample peak area; $C_{\mathrm{st}}$ is the saccharide standard concentration $\mathrm{mg} / \mathrm{ml} ; V$ is the volume in which the sample was prepared, $\mathrm{ml} ; S_{\mathrm{st}}$ is the saccharide standard peak area; $m$ is the sample weight, $\mathrm{g}$.
HPLC operating conditions Mobile phase flow rate $1.2 \mathrm{ml} / \mathrm{min}$; injection volume of the sample $-20 \mu \mathrm{l}$; column temperature $-28^{\circ} \mathrm{C}$; an evaporative light scattering detector, isocratic elution, a mixture of acetonitrile and water (75:25, $\mathrm{v}$ : v) was used as the mobile phase.

\section{Principal component analysis}

The PCA was used for the spectral data clustering to evaluate the differences among the SERS spectra of the bilberries. PCA analysis in a spectral range from 250 to $1800 \mathrm{~cm}^{-1}$ was carried out in the Matlab platform using Raman preprocessing software. The normalized SERS spectra of the bilberries of each country were grouped and the PCA scores were plotted. PCA score distributions were used to distinguish the spectral properties contributing to the spectral variance.

\section{Results and discussion}

Raman spectroscopy is a non-sensitive technique related to a weak scattering because only one of a million photons are scattered in-elastically. As a result, a low-intensity Raman bands are observed in the spectrum leading to the complicated analysis of the test object. Especially in the cases, when the concentrations of the particular molecules are low. Moreover, during the analysis of the materials that contain pigments, there is a probability that the fluorescent background will be observed in the spectra that will affect the qualitative as well as quantitative analysis of the sample. Given that the bilberries are rich in anthocyanin, the main peaks expressed in the spectra belong to the molecular vibrations of these phenolic compounds. Anthocyanins are pigments that give fruits, berries and vegetables a beautiful color varying from blue to red. However, the presence of anthocyanin usually determines a strong fluorescent background in Raman spectra as it is shown in Fig. 1A. As seen, the fluorescence is so intense that it suppresses a weak Raman signal emitted by the pigment. For this reason, it becomes impossible to distinguish the peaks. To avoid this issue, SERS approach was applied for the analysis of the bilberry extracts.

The principle of the SERS technique can be described as a phenomenon when the analyte adsorbs on the nanostructured metal surface leading to the enhanced intensity of Raman signal. Moreover, the fluorescence quenching occurs after the adsorbance of the analyte takes place on the surface of the nanoparticles [26]. This phenomenon is caused by the energy transfer occurring between the nanostructured metal and the fluorophore. Therefore, to perform the SERS measurements of the bilberry extracts, silver SERS substrates were prepared and analyzed. As seen in Fig. 1C, the morphological analysis of the SERS substrate showed 


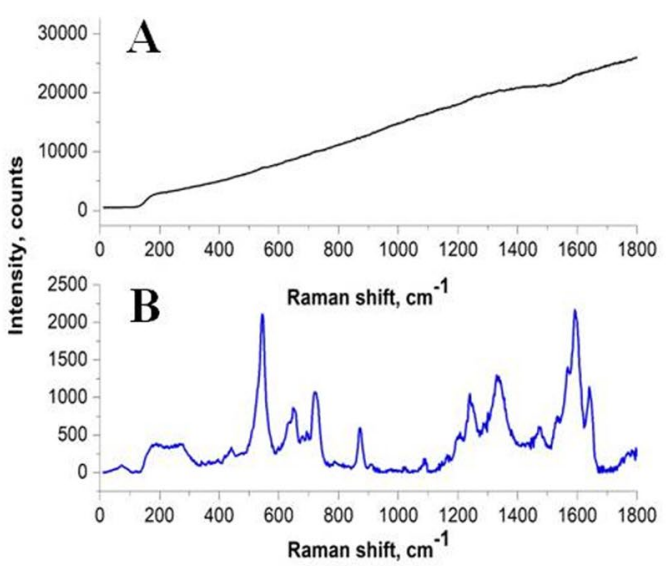

Fig. 1 A Raman spectra of the bilberries recorded on the blank silicon wafer; B SERS spectra of the bilberries recorded on the nanostructured silver SERS substrate; C SEM image of the silver SERS

that the nanoparticles were successfully deposited on the silicon wafers. In addition, the EDX analysis of the SERS substrate was carried out that confirmed the presence of the silver (Fig. 1D). Random arrangement and distribution of the metal nanoparticles ensure a high density of the plasmonic hot spots- the nanometric gaps (1-2 nm) between the nanoparticles. Therefore, when the analyte is "entrapped" into the hot spot and irradiated with a laser beam, a strong electromagnetic field is generated inside the nano-gap resulting in the increased intensity of the peaks. The quantitative parameter describing the ability of the SERS substrate to enhance the Raman signal of the analyte is the enhancement factor. In this work, the EF was determined by evaporating pentacene monolayer on the nanostructured silver surface. As seen in Fig. S2 (see Supplementary Material), the pentacene was almost undetectable on the blank silicon wafer. Meanwhile, silver SERS substrate allowed to obtain the intense Raman signals. Based on the results of pentacene measurements, the EF of the synthesized silver SERS substrates was found to be $10^{5}$. The sensitive SERS substrates were further applied for the measurements of the bilberry extracts. As seen in Fig. 1B, the bands of the bilberries are well expressed in the full range of the spectra.

After the evaluation of the morphological and sensitivity properties of the nanostructured silver substrates, SERS measurements were carried out for the wild bilberries growing in the particular regions in Lithuania (LTU), Latvia (LVA), Norway (NOR) and Finland (FNL). The results are presented in Fig. 2A.

Figure 2B shows the set of the bilberries SERS spectra of each country (first replicate). At the glance, the spectra

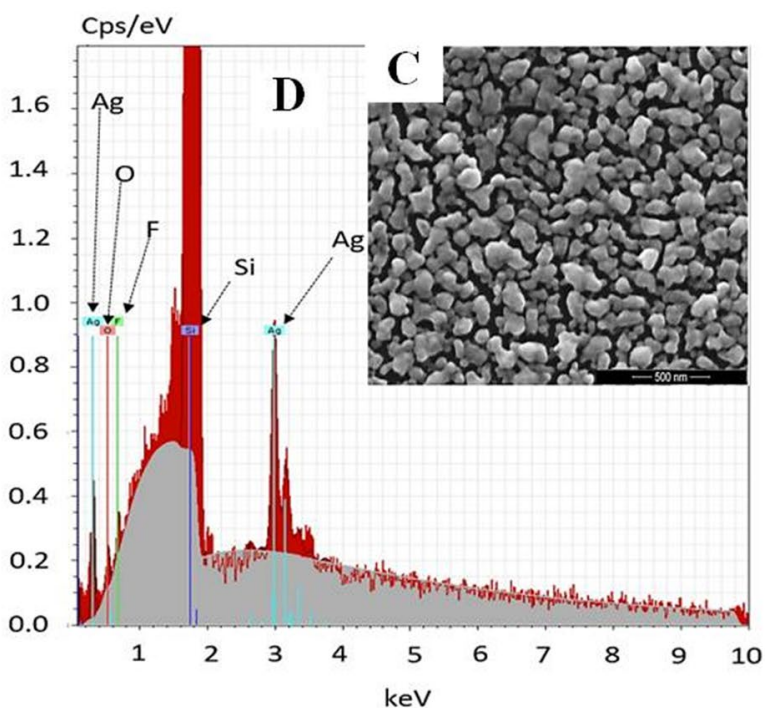

substrate (magnification: $\times 100,000$; high vacuum mode $30.000 \mathrm{kV}$ ); D EDX spectra of the silver SERS substrate

are very similar due to the multiple overlapping bands that belong to a number of molecules in the sample. Therefore, to perform a precise analysis of the spectra, the deconvolution algorithm (Gaussian) was applied to fit the peaks. As a model for fitting, the averaged SERS spectrum of the Lithuanian bilberries was selected. The fitted bands were numbered and the vibrational assignments were provided in Table 1. As seen in the deconvoluted SERS spectra (Fig. 3), the most intensive bands arise from the vibrational modes of the most abundant anthocyanin found in bilberriesdelphinidins, cyanidins, petunidins, malvidins, pelargonidins and peonidins $[15,16,27,28]$. The intensive peaks located at $\sim 1530 \mathrm{~cm}^{-1}, \sim 1590 \mathrm{~cm}^{-1}$, and $\sim 1640 \mathrm{~cm}^{-1}$ are related to the ring stretching vibrations and a band observed at $\sim 1243 \mathrm{~cm}^{-1}$ belongs to the stretching mode of $\mathrm{C}-\mathrm{OH}$ bond in anthocyanin [16]. At low $\mathrm{pH}$ value ( 4.0), the spectral pattern of the bilberry extracts is determined by the vibrations of the glycosylated anthocyanin.

The vitamin $\mathrm{C}$ related vibrations are observed at $\sim 467 \mathrm{~cm}^{-1}$ and $\sim 1758 \mathrm{~cm}^{-1}$ [29]. Meanwhile, the peaks located at $\sim 1590 \mathrm{~cm}^{-1}$ and $\sim 794, \sim 1162 \mathrm{~cm}^{-1}$ belong to retinol and phenolic acids, respectively [27, 30]. Bilberries contain a significant amount of polyunsaturated essential fatty acids (i.e., linoleic and linolenic acids) [31] and exhibit a $\mathrm{C}=\mathrm{O}$ stretching vibrations at $\sim 1744 \mathrm{~cm}^{-1}$ and $\sim 1771 \mathrm{~cm}^{-1}$ [32]. Given that glutamic acid and valine are the dominant amino acids in bilberries [33], they also generate SERS signals at $\sim 902 \mathrm{~cm}^{-1}, \sim 1275 \mathrm{~cm}^{-1}$, and $\sim 1562 \mathrm{~cm}^{-1}$ [34]. Other important compounds present in bilberries are carbohydrates that include monosaccharides (fructose, glucose) and polysaccharides (fibers). Therefore, the peaks 
A
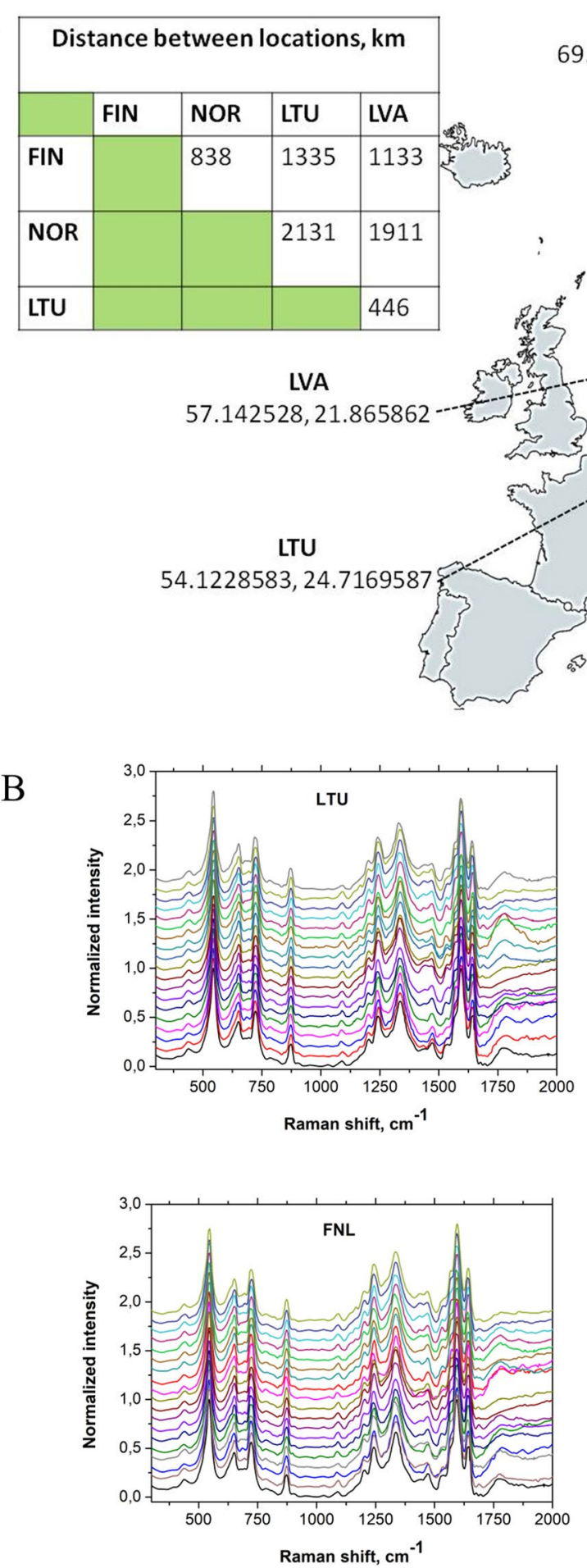

NOR

$69.6944201,18.9911423$

FNL

64.516904, 26.422660
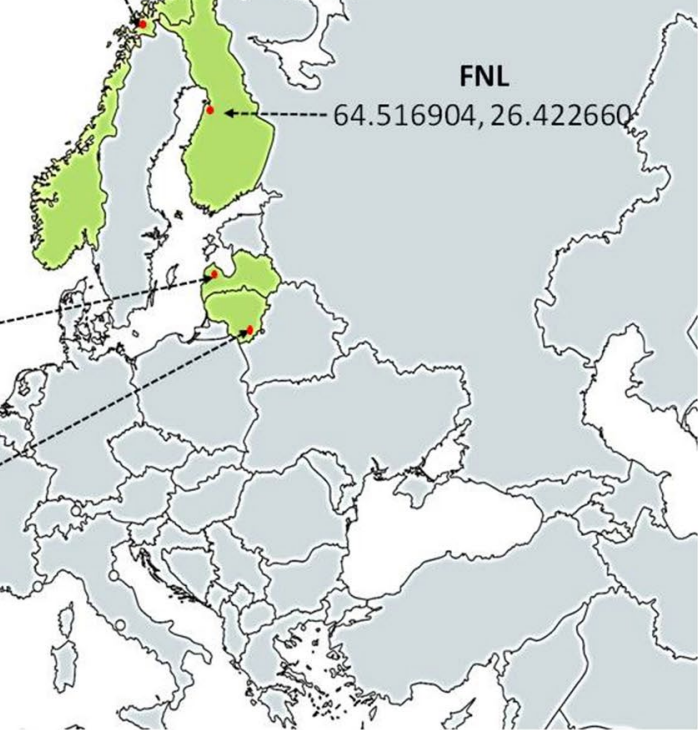

$\hat{n}$
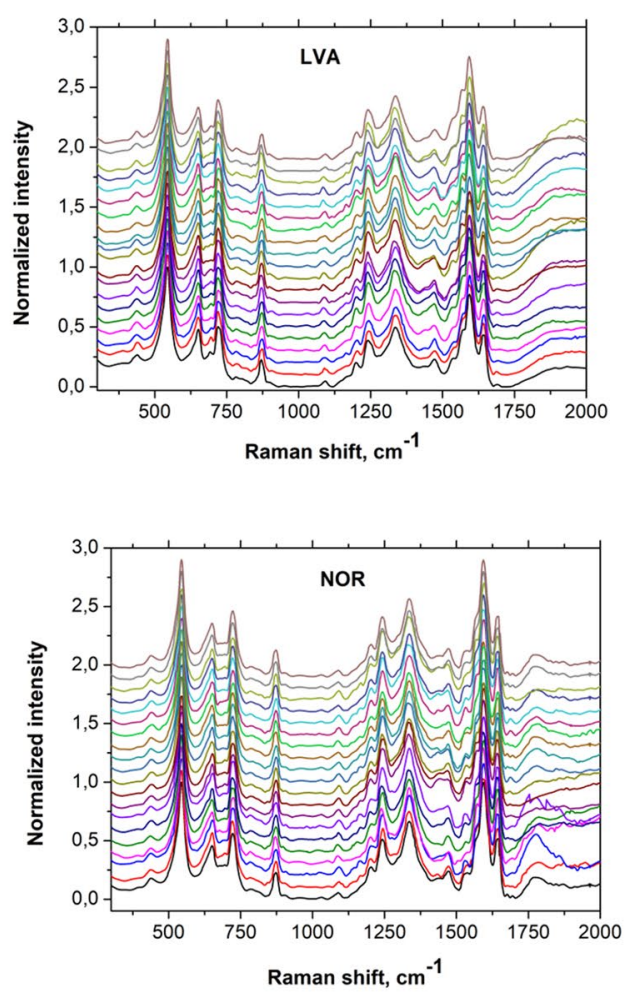

Fig. 2 A The geographical coordinates of the regions in Lithuania, Latvia, Norway and Finland where the wild bilberries were harvested; $\mathbf{B}$ the set of the bilberries SERS spectra of each country recorded at 20 different points of the sample

located at $\sim 423 \mathrm{~cm}^{-1}, \sim 730 \mathrm{~cm}^{-1}$, and $\sim 1204 \mathrm{~cm}^{-1}$ and at $\sim 1142 \mathrm{~cm}^{-1}$ and $\sim 1471 \mathrm{~cm}^{-1}$ belong to D-glucose and D-fructose, respectively. Based on the analysis of the deconvoluted spectrum, the comparison of the series of the bilberries SERS spectra was carried out (Fig. 2B). Despite the visual similarity of the SERS spectral pattern, the spectral clusters of each country were observed in PCA plot. As seen in Fig. 4A, the highest spectral similarity was common 
Table 1 Assignment of the observed SERS bands

\begin{tabular}{|c|c|c|c|}
\hline Peak number & Peak position, $\mathrm{cm}^{-1}$ & Assignment & Source \\
\hline 1 & 423 & D-Glucose & {$[35]$} \\
\hline 2 & 467 & Vitamin $\mathrm{C}, \mathrm{CO}$ in plane deformation & [29] \\
\hline 3 & 539 & Cyanidin, $\mathrm{CC}$, in plane bending & [15] \\
\hline 4 & 542 & Malvidin, $\mathrm{CC}$ in plane bending & \\
\hline 5 & 645 & Malvidin & \\
\hline 6 & 651 & Peonidin & \\
\hline 7 & 730 & D-Glucose & {$[35]$} \\
\hline 8 & 794 & Chlorogenic acid & {$[30]$} \\
\hline 9 & 875 & Pelargonidin, $\mathrm{CH}$, cyanidin, $\mathrm{CH}$, out of plane bending & {$[15]$} \\
\hline 10 & 902 & Amino acids & {$[34]$} \\
\hline 11 & 1082 & Petunidin, delphinidin, aromatic $\mathrm{CH}$ bending and rocking, $\mathrm{C}-\mathrm{OH}$ bending & {$[15]$} \\
\hline 12 & 1142 & D-Fructose & [35] \\
\hline 13 & 1162 & Phenolic acids & {$[30]$} \\
\hline 14 & 1204 & D-Glucose & [35] \\
\hline 15 & 1243 & $\mathrm{C}-\mathrm{OH}$, malvidins & {$[15,16]$} \\
\hline 16 & 1275 & Amino acids & [34] \\
\hline 17 & 1341 & Delphinidin, $\mathrm{CC}$ stretching (interring), $\mathrm{CH}$ in plane bending & {$[15]$} \\
\hline 18 & 1439 & D-Fructose & {$[35]$} \\
\hline 19 & 1471 & D-Fructose & \\
\hline 20 & 1530 & Petunidins, ring stretching & {$[15,16]$} \\
\hline 21 & 1562 & Amino acids & {$[34]$} \\
\hline 22 & 1590 & Petunidins, peonidins, malvidins, ring stretching, retinol, $\mathrm{C}=\mathrm{C}$ & {$[15,16,27]$} \\
\hline 23 & 1643 & Pelargonidins, peonidins, ring stretching & {$[15,16]$} \\
\hline 24 & 1744 & Fatty acids, $\mathrm{C}=\mathrm{O}$ stretching, pectins & {$[32]$} \\
\hline 25 & 1758 & Vitamin $\mathrm{C}, \mathrm{C}=\mathrm{O}$ stretching & [29] \\
\hline 26 & 1771 & Linoleic acid, $\mathrm{C}=\mathrm{O}$ & {$[32]$} \\
\hline
\end{tabular}

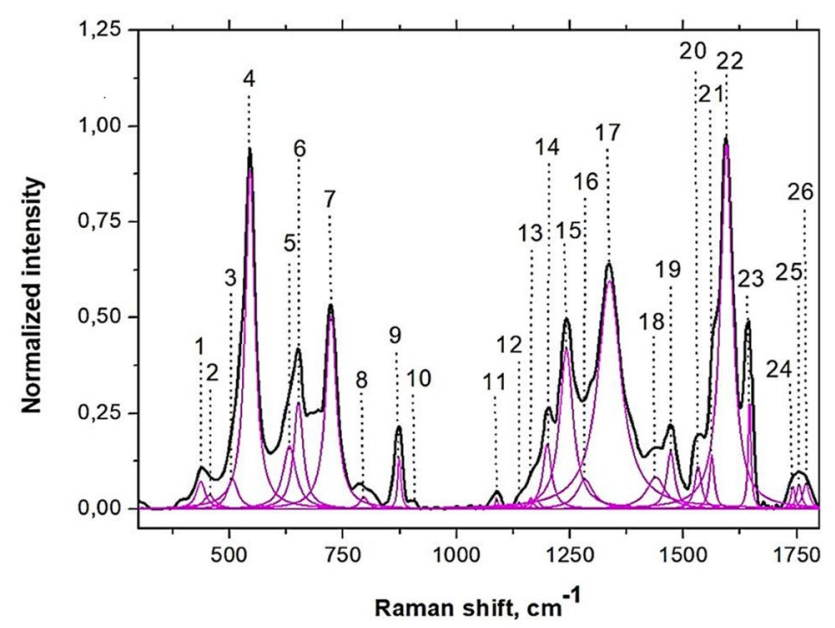

Fig. 3 The deconvoluted average SERS spectrum of Lithuanian bilberries

for the Norwegian and Finnish bilberries. Similar results were common for the second and the third experimental replicates (see Figs. S3 and S4, supplementary material).
Such similarity is determined by the similar geographical situation-both countries are located in the Northern Europe. According to the previous research, a significantly higher content of total anthocyanin is abundant in bilberries of Northern regions [36]. It is related to the lower temperatures at which the accumulation of anthocyanin increases [24]. In order to simplify the analysis and to determine the main spectral differences leading to the separation of the spectral groups in PCA plot, all the SERS spectra of each country were averaged and plotted on the same graph (Fig. 4B). It was noticed that the different qualitative/quantitative content of anthocyanin reflects in the SERS spectra. As seen in Fig. 4B, the vibrations at $\sim 645 \mathrm{~cm}^{-1}$ and $\sim 651 \mathrm{~cm}^{-1}$ correspond to anthocyanin and are a slightly shifted in the case of Finnish and Norwegian bilberries. Moreover, a band located at $\sim 1341 \mathrm{~cm}^{-1}$ also belongs to anthocyanin and is of higher intensity in the case of Northern bilberries. These observations suggest that the Finnish and Norwegian bilberries contain higher amount of anthocyanin compared to the bilberries growing on the Eastern shore of the Baltic Sea. Another band which significantly differs in shape and intensity is located at $\sim 1471 \mathrm{~cm}^{-1}$ and corresponds to D-fructose. 

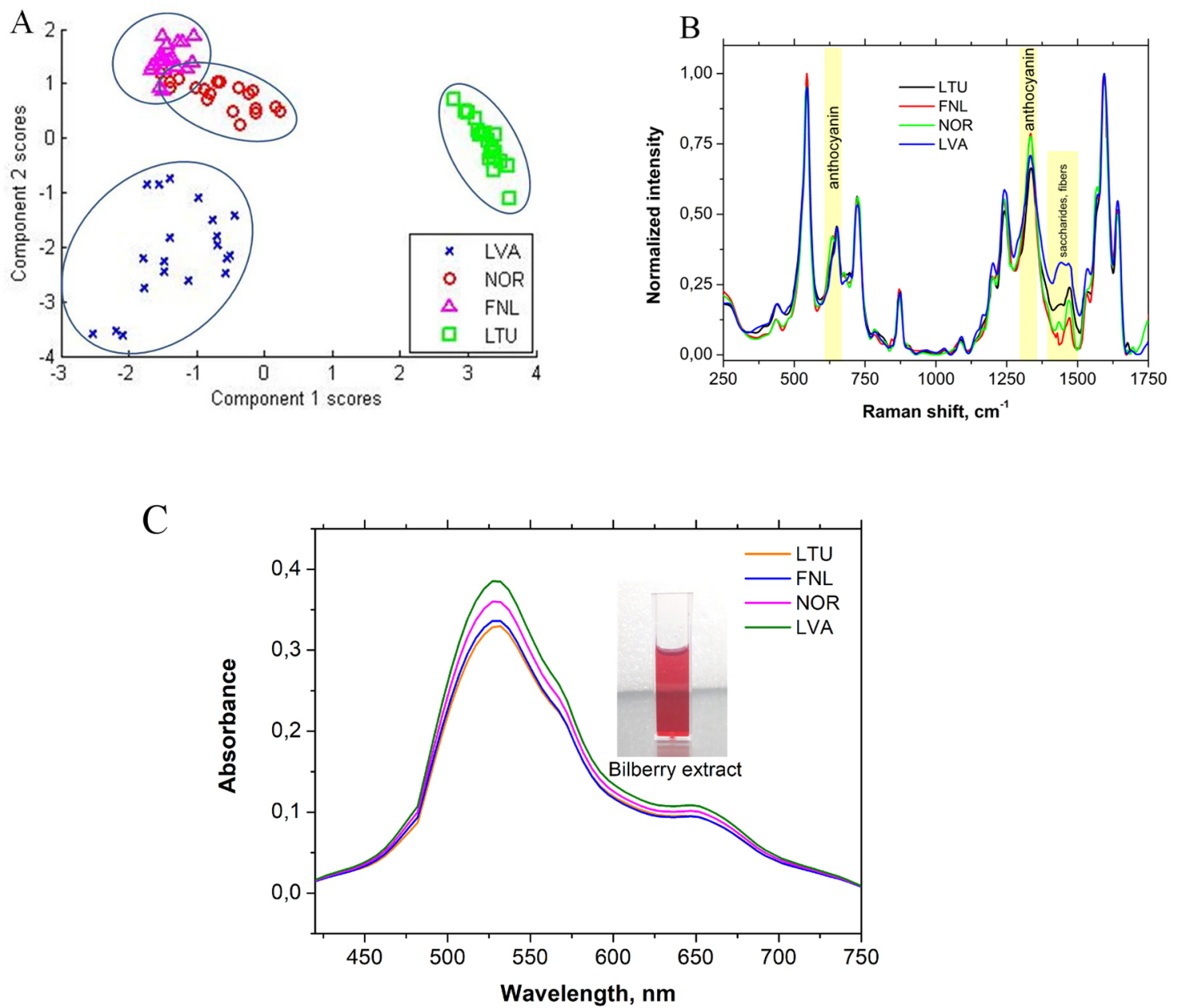

Fig. 4 A PCA score plot that shows the spectral discrimination between groups; $\mathbf{B}$ the averaged SERS spectra of bilberries of each country; $\mathbf{C}$ UV-Vis spectra of the bilberry extracts

The predominant saccharides in bilberries are fructose and glucose, while sucrose is found only at trace amounts due to the enzymatic hydrolysis of disaccharide [37]. The broadened peak arising from the vibrations of D-fructose leads to the hypothesis that Lithuanian and Latvian bilberries contain higher amount of fructose compared to those growing in Finland and Norway.

To confirm the discussed hypotheses, the determination of the total anthocyanin content in bilberries was carried out by UV-Vis spectroscopy. As seen in Fig. 4C, the highest absorbance was common for the Finnish and Norwegian bilberries. The calculated quantities of anthocyanin were found to be $445,459,508$ and $525 \mathrm{mg} / 100 \mathrm{~g}$ for Lithuanian, Latvian, Norwegian and Finnish berries, respectively (see Table 2).

HPLC analysis of the saccharides revealed that the highest amount of D-fructose (4.71\%) is common for Lithuanian bilberries. A similar amount (4.25\%) was found in Latvian berries. Meanwhile, Norwegian and Finnish bilberries contained $3.83 \%$ and $3.72 \%$ of D-fructose, respectively. These findings are consistent with the SERS experimental results and confirm the applicability of the SERS-PCA method for the determination of the geographical origin of the bilberries.

\section{Conclusions}

The wild bilberries growing at the different geographical locations of the Baltic-Nordic regions were investigated by the SERS. Silver nanoparticles based SERS substrates allowed to quench the fluorescence background, to enhance the Raman signal and to distinguish the characteristic Raman signals of the compounds present in bilberries. To evaluate the differences between spectral data of the bilberries, the chemometric analysis was performed. It was found that SERS approach in combination with PCA enables to distinguish bilberries growing in the different countries. It was 
Table 2 The amount of the saccharides and anthocyanin found in Lithuanian, Latvian, Norwegian and Finnish bilberries

\begin{tabular}{llllll}
\hline Country & D-Glucose & D-Fructose & Sucrose & $\begin{array}{l}\text { Total values of sac- } \\
\text { charides, \% (HPLC) }\end{array}$ & $\begin{array}{l}\text { Total content of } \\
\text { anthocyanin, mg/100 g } \\
\text { (UV-Vis) }\end{array}$ \\
\hline LTU & 3.50 & 4.71 & 0.00 & 8.21 & 445 \\
LVA & 3.11 & 4.25 & 0.01 & 7.36 & 459 \\
NOR & 3.15 & 3.83 & 0.00 & 6.98 & 508 \\
FNL & 2.93 & 3.72 & 0.00 & 6.65 & 525 \\
\hline
\end{tabular}

determined that significant differences in the averaged and normalized SERS spectra were determined by the qualitative and quantitative compositions of anthocyanin and saccharides. However, it is possible that other components such as amino acids, vitamins, phenolic and fatty acids, also contributed to the clusterization of the groups in the PCA plot. The proposed SERS-PCA approach was found to be fast, sensitive and cost-effective for the analysis of the bilberries at the molecular level. Therefore, the systematic collection of the SERS spectral libraries opens up opportunities for the rapid evaluation of the bilberries' geographical origin.

Supplementary Information The online version contains supplementary material available at https://doi.org/10.1007/s00217-021-03887-8.

Acknowledgements This work was supported by the Interreg Baltic Sea Region Program. Project title: Market-driven authentic Non-Timber Forest Products from the Baltic region-focus on wild and semi cultivated species with business potential (NovelBaltic), Project No. R079. The authors would like to thank Dr. Darius Cernauskas and Ernestas Mockus for HPLC analysis of the saccharides in bilberries.

Author contributions LT: conceptualization, methodology, investigation, analysis, and writing an original draft. VS: measurements, investigation, analysis, writing an original draft, review and editing.

\section{Declarations}

Competing interest The authors declare no competing interests.

Compliance with ethics requirements This article does not contain any studies with human participants or animals performed by any of the authors.

Open Access This article is licensed under a Creative Commons Attribution 4.0 International License, which permits use, sharing, adaptation, distribution and reproduction in any medium or format, as long as you give appropriate credit to the original author(s) and the source, provide a link to the Creative Commons licence, and indicate if changes were made. The images or other third party material in this article are included in the article's Creative Commons licence, unless indicated otherwise in a credit line to the material. If material is not included in the article's Creative Commons licence and your intended use is not permitted by statutory regulation or exceeds the permitted use, you will need to obtain permission directly from the copyright holder. To view a copy of this licence, visit http://creativecommons.org/licenses/by/4.0/.

\section{References}

1. Zheng J, He L (2014) Surface enhanced Raman spectroscopy for the chemical analysis of food. Comp Rev Food Sci F 13:317-328

2. Gillibert R, Huang JQ, Zhang Y, Fu WL, de la Chapelle ML (2018) Food quality control by surface enhanced Raman scattering. TrAC 105:185-190

3. Xu ML, Gao Y, Han XX, Zhao B (2017) Detection of pesticide residues in food using surface-enhanced Raman spectroscopy: a review. J Agric Food Chem 65:6719-6726

4. Dhakal S, Chao K, Huang Q, Kim M, Schmidt W, Qin J, Broadhurst CL (2018) A simple surface-enhanced Raman spectroscopic method for on-site screening of tetracycline residue in whole milk. Sensors. https://doi.org/10.3390/s18020424

5. Muhammad M, Yan B, Yao G, Chao K, Zhu C, Huang Q (2020) Surface-enhanced Raman spectroscopy for trace detection of tetracycline and dicyandiamide in milk using transparent $\mathrm{Ag}$ nanoparticle arrays. ACS Appl Nano Mater. https://doi.org/10. 1021/acsanm.0c01389

6. Bodelón G, Pastoriza-Santos I (2020) Recent Progress in Surface-Enhanced Raman Scattering for the Detection of Chemical Contaminants in Water. Front Chem. https://doi.org/10.3389/ fchem. 2020.00478

7. Wei C, Li M, Zhao X (2018) Surface-enhanced Raman scattering (SERS) with silver nano substrates synthesized by microwave for rapid detection of foodborne pathogens. Front Microbiol. https://doi.org/10.3389/fmicb.2018.02857

8. Witkowska E, Korsak D, Kowalska A, Księżopolska-Gocalska M, Niedziółka-Jönsson J, Roźniecka E, Michałowicz W, Albrycht P, Podrażka M, Hołyst R, Waluk J, Kamińska A (2017) Surface-enhanced Raman spectroscopy introduced into the International Standard Organization (ISO) regulations as an alternative method for detection and identification of pathogens in the food industry. Anal Bioanal Chem 409:1555-1567

9. Abid Hasan SM, He Y, Chang TW, Wang J, Gartia MR (2018) Detecting DNA methylation using surface-enhanced Raman spectroscopy. J Phys Chem C 123:698-709

10. Myintzu Hlaing M, Wood B, McNaughton D, Ying D, Augustin MA (2017) Raman spectroscopic analysis of Lactobacillus rhamnosus GG in response to dehydration reveals DNA conformation changes. J Biophotonics 10:589-597

11. Li D, Meng X, Li B (2016) Profiling of anthocyanins from blueberries produced in China using HPLC-DAD-MS and exploratory analysis by principal component analysis. J Food Compost Anal 47:1-7

12. Nadulski R, Masłowski A, Mazurek A, Sobczak P, Szmigielski M, Żukiewicz-Sobczak W, Niedziółka I, Mazur J (2019) Vitamin $\mathrm{C}$ and lutein content of northern highbush blueberry (Vaccinium corymbosum L.) juice processed using freezing and thawing. J Food Meas Charact 13:2521-2528

13. Schulz H, Baranska M (2007) Identification and quantification of valuable plant substances by IR and Raman spectroscopy. Vib Spectrosc 43:13-25 
14. Wu L, Yan H, Li G, Xu X, Zhu L, Chen X, Wang J (2019) Surface-imprinted gold nanoparticle-based surface-enhanced Raman scattering for sensitive and specific detection of patulin in food samples. Food Anal Methods 12:1648-1657

15. Zaffino C, Russo B, Bruni S (2015) Surface-enhanced Raman scattering (SERS) study of anthocyanidins. Spectrochim Acta A Mol Biomol Spectrosc 149:41-47

16. Zaffino C, Bruni S, Russo B, Pilu R, Lago C, Colonna GM (2016) Identification of anthocyanins in plant sources and textiles by surface enhanced Raman spectroscopy (SERS). J Raman Spectrosc 47:269-276

17. Castañeda-Ovando A, de Lourdes P-H, Páez-Hernández ME, Rodríguez JA, Galán-Vidal CA (2009) Chemical studies of anthocyanins: a review. Food Chem 113:859-871

18. Nieuwoudt MK, Holroyd SE, McGoverin CM, Simpson MC, Williams DE (2016) Raman spectroscopy as an effective screening method for detecting adulteration of milk with small nitrogen-rich molecules and sucrose. JDS 99:2520-2536

19. Salo HM, Nguyen N, Alakärppä E, Klavins L, Hykkerud AL, Karppinen K, Jaakola L, Klavins M, Häggman H (2021) Authentication of berries and berry-based food products. Compr Rev Food Sci 20:5197-5225

20. Bertoldi D, Cossignani L, Blasi F, Perini M, Barbero A, Pianezze S, Montesano D (2019) Characterisation and geographical traceability of Italian goji berries. Food chem 27:5585-5593

21. Penman KG, Halstead CW, Matthias A, De Voss JJ, Stuthe JM, Bone KM, Lehmann RP (2006) Bilberry adulteration using the food dye amaranth. J Agric Food Chem 54:7378-7382

22. Foster S, Blumenthal M (2012) The adulteration of commercial bilberry extracts. HerbalGram 96:64-73

23. He H, Li P, Tang X, Lin D, Xie A, Shen Y, Yang L (2019) Developing cysteamine-modified SERS substrate for detection of acidic pigment with weak surface affinity. Spectrochim Acta A Mol Biomol Spectrosc 212:293-299

24. Spinardi A, Cola G, Gardana CS, Mignani I (2019) Variation of anthocyanins content and profile throughout fruit development and ripening of highbush blueberry cultivars grown at two different altitudes. Front Plant Sci. https://doi.org/10.3389/fpls.2019. 01045

25. European pharmacopoeia (2005) Fifth Edition. Volume 2. Strasbourg: Council of Europe, pp 1099-1100

26. Shan F, Zhang XY, Fu XC, Zhang LJ, Su D, Wang SJ, Wu JY, Zhang T (2017) Investigation of simultaneously existed Raman scattering enhancement and inhibiting fluorescence using surface modified gold nanostars as SERS probes. Sci Rep 7:1-10
27. Failloux N, Bonnet I, Baron MH, Perrier E (2003) Quantitative analysis of vitamin A degradation by Raman spectroscopy. Appl Spectrosc 57:1117-1122

28. Zheng X, Hu Y, Anggreani E, Lu X (2017) Determination of total phenolic content and antioxidant capacity of blueberries using Fourier transformed infrared (FT-IR) spectroscopy and Raman spectroscopy. J Food Meas Charact 11:1909-1918

29. Panicker CY, Varghese HT, Philip D (2006) FT-IR, FT-Raman and SERS spectra of Vitamin C. Spectrochim Acta A Mol Biomol Spectrosc 65:802-804

30. Pompeu DR, Larondelle Y, Rogez H, Abbas O, Pierna JAF, Baeten V (2018) Characterization and discrimination of phenolic compounds using Fourier transform Raman spectroscopy and chemometric tools. Base. https://doi.org/10.25518/1780-4507.16270

31. Bunea A, Rugină D, Pintea A, Andrei S, Bunea C, Pop R, Bele C (2012) Carotenoid and fatty acid profiles of bilberries and cultivated blueberries from Romania. Chem Pap 66:935-939

32. Machado NFL, de Carvalho LB, Otero JC, Marques MPM (2012) The autooxidation process in linoleic acid screened by Raman spectroscopy. J Raman Spectrosc 43:1991-2000

33. Padmanabhan P, Correa-Betanzo J, Paliyath G (2016) Berries and related fruits. Encycl Food Health. https://doi.org/10.1016/B9780-12-384947-2.00060-X

34. Zhu G, Zhu X, Fan Q, Wan X (2011) Raman spectra of amino acids and their aqueous solutions. Spectrochim Acta A Mol Biomol Spectrosc 78:1187-1195

35. Wiercigroch E, Szafraniec E, Czamara K, Pacia MZ, Majzner K, Kochan K, Kaczor A, Baranska M, Malek K (2017) Raman and infrared spectroscopy of carbohydrates: a review. Spectrochim Acta A Mol Biomol Spectrosc 185:317-335

36. Lätti AK, Riihinen KR, Kainulainen PS (2008) Analysis of anthocyanin variation in wild populations of bilberry (Vaccinium myrtillus L.) in Finland. J Sci Food Agric 56:190-196

37. Mikulic Petkovsek M, Schmitzer V, Slatnar A, Stampar F, Veberic $\mathrm{R}$ (2015) A comparison of fruit quality parameters of wild bilberry (Vaccinium myrtillus L.) growing at different locations. J Sci Food Agric. https://doi.org/10.1002/jsfa.6897

Publisher's Note Springer Nature remains neutral with regard to jurisdictional claims in published maps and institutional affiliations. 